\title{
Consumo de aves en el valle medio del río Negro durante el Holoceno tardío final: los restos del sitio Colforta 1 (provincia de Río Negro, Argentina)
}

\author{
Emiliano Mange, Maitén Di Lorenzo, Lucio González Venanzi e Inés Anthonioz Blanc \\ Recibido 06 de febrero 2021. Aceptado 22 de abril 2021
}

\begin{abstract}
RESUMEN
Se presenta el análisis de los restos óseos y de las cáscaras de huevo de aves del sitio arqueológico Colforta 1, ubicado en el valle medio del río Negro (Norpatagonia, Argentina), con el objetivo de determinar cuáles fueron los agentes responsables de su acumulación. El conjunto habría sido depositado principalmente por las actividades antrópicas vinculadas con el consumo. El grupo más capturado fue el de los tinámidos, familia de aves medianas terrestres, especialmente martinetas (Eudromia elegans), aunque también se aprovecharon especies de diferentes tamaños y hábitos. Además, se habrían recolectado huevos de diversas especies, incluyendo rheidos y tinámidos. Se discute el aprovechamiento y el procesamiento antrópico de estos recursos en el sitio y su importancia en los valles fluviales de Norpatagonia durante el Holoceno tardío para las sociedades cazadoras-recolectoras.
\end{abstract}

Palabras clave: Cazadores-recolectores; Aves; Norpatagonia, Holoceno tardío.

\section{Bird consumption in the middle Negro River valley during the Final Late Holocene: The remains of the Colforta 1 site (Río Negro Province, Argentina)}

\begin{abstract}
This paper presents the analysis of the bone remains and the eggshells of birds from Colforta 1 archaeological site, located in the middle Negro River valley (Northern Patagonia, Argentina), with the objective of determining the agents responsible for their accumulation. The analysis carried out shows that a large part of the sample would have been deposited as a result of anthropic activities. The main group of birds captured were tinamous, a family of medium-sized terrestrial birds, although species of different sizes, habits and environments were also exploited. In addition, the eggs of various species, including rheids and tinamous, would have been collected seasonally. The anthropic processing of avifaunistic resources on the site and their importance in the Norpatagonia river valleys for late Holocene huntergatherer societies are discussed.
\end{abstract}

Keywords: Hunter-gatherers; Birds; Northern Patagonia, Late Holocene.

\footnotetext{
Emiliano Mange. Consejo Nacional de Investigaciones Científicas y Técnicas (CONICET). Facultad de Ciencias Naturales y Museo, Universidad Nacional de La Plata (FCNyM-UNLP), Argentina. Anexo Museo, Oficina I28, Avenida 122 y 60 (CP 1900), La Plata. E-mail: emilianomange@gmail.com

Maitén Di Lorenzo. CONICET. Instituto de Investigaciones en Diversidad Cultural y Procesos del Cambio, Universidad Nacional de Río Negro (UNRN), Argentina. Bartolomé Mitre 630 (CP 8400), San Carlos de Bariloche. E-mail: maiten.dilorenzo@gmail.com

Lucio González Venanzi. CONICET. FCNyM-UNLP, Argentina. Anexo Museo, Oficina I28, Avenida 122 y 60 (CP I900), La Plata. E-mail: luciogonzalezvenanzi@gmail.com

Inés Anthonioz Blanc. FCNyM-UNLP, Argentina. Anexo Museo, Oficina 128, Avenida 122 y 60 (CP 1900), La Plata. E-mail: inesanthoniozblanc@gmail.com
}

Intersecciones en Antropología 22(2), julio-diciembre: 169-181. 2021. ISSN-e 1850-373X

https://doi.org/10.37176/iea.22.2.2021.617

Facultad de Ciencias Sociales - UNICEN - Argentina 


\section{INTRODUCCIÓN}

Los estudios arqueofaunísticos en la Patagonia argentina se centraron en el análisis de grandes vertebrados, principalmente guanaco (Lama guanicoe), ñandú (Rhea sp.) y mamíferos marinos (pinnípedos), mientras que los vertebrados medianos y pequeños ocuparon un lugar periférico en las discusiones vinculadas con la subsistencia (Cruz et al., 2007). La falta de interés por los pequeños vertebrados hasta la década de 1980 puede vincularse con los marcos teóricos dominantes (principalmente culturalistas) y con metodologías de recuperación y análisis inadecuadas (Alcaráz, 2017; Fernández et al., 2017). Con el advenimiento de la Nueva Arqueología, se produjo un renovado interés por los estudios faunísticos (Politis, 2003) y se incluyó en la agenda de investigación el análisis de restos de microvertebrados. En este contexto se realizaron estudios arqueofaunísticos en las grandes cuencas fluviales de Norpatagonia desde las últimas décadas del siglo XX, algunos de ellos relacionados con los pequeños vertebrados (e.g., Bond et al., 1981;
Tonni, 1981). Posteriormente, desde comienzos del siglo XXI se realizaron investigaciones arqueológicas regionales en la costa del golfo San Matías (Borella et al., 2015; Favier Dubois et al., 2016), en el valle inferior del río Colorado (Martínez, 2008-2009, 2015) y en los sectores medios de los valles de los ríos Negro (Prates, 2008; Mange, 2019) y Limay (Crivelli Montero, 2010) (Figura 1). En el marco de estos proyectos se realizaron numerosos trabajos arqueofaunísticos que han permitido profundizar en el conocimiento del consumo de pequeños animales y de la amplitud de la dieta (Prates, 2008; Alcaráz, 2015, 2017; Mange et al., 2016; Fernández et al., 2017). Además, en algunas de estas contribuciones se discutió el aprovechamiento de aves (Prates y Acosta Hospitaleche, 2010; Marani y Borella, 2014; Alcaráz, 2015, 2017).

En los últimos años se excavaron nuevos sitios arqueológicos en el valle medio del río Negro y se realizaron estudios faunísticos (Mange et al., 2016; Mange, 2019). Uno de los sitios estudiados es Colforta 1 (en adelante, Co1), ubicado en cercanías

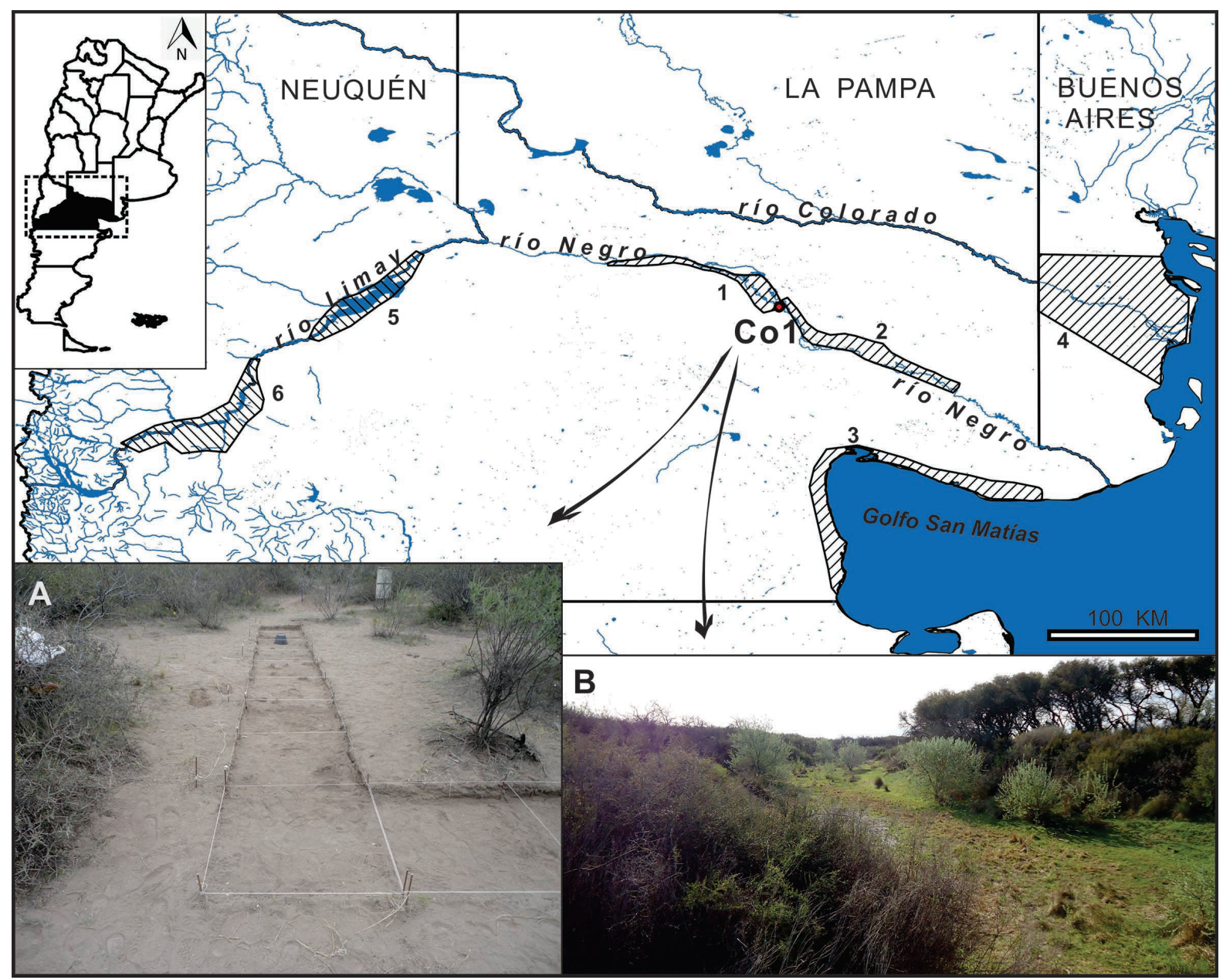

Figura 1. Ubicación del sitio Colforta 1 (Co1) y regiones mencionadas en el trabajo: sectores medio/superior (1) y medio/inferior del valle del río Negro (2), costa del golfo San Matías (3), valle inferior del río Colorado (4) y sectores medio/inferior (5) y medio/ superior (6) del río Limay. Fotografías: (A) cuadrículas excavadas sobre el médano y (B) laguna en el paleocauce cercano. 
del extremo sureste de la isla Grande de Choele Choel (39³2'09"S; 6533'29"O, Figura 1), sobre un médano adyacente a un paleocauce que en este sector presenta una laguna, actualmente seca. En la superficie del médano y en posición estratigráfica se hallaron diversos artefactos (líticos, cerámicos, vegetales, etc.) y una gran variedad y densidad de restos faunísticos, que indican que habría sido un campamento residencial de actividades múltiples (Mange, 2019). El fechado radiocarbónico obtenido a partir de un conjunto de restos de carbones vegetales (790 $\pm 50 \mathrm{AP}, \mathrm{LP}-3493)$ ubica la ocupación en el Holoceno tardío final. La calibración realizada con el programa Calib Rev 7.0.4 (Stuiver et al., 2017) indica que el fechado corresponde al intervalo 633-761 años AP (91,7\% de probabilidad). Los materiales faunísticos incluyen valvas de moluscos, cáscaras de huevos de aves y restos óseos principalmente de mamíferos, peces y aves, e indican la captura y el consumo de diversos recursos en las lagunas y cauces cercanos al sitio y en el interior del valle (Mange, 2019). Los materiales presentan similitudes generales con otros conjuntos arqueofaunísticos del valle medio del río Negro, caracterizados por el consumo de diversos recursos (Prates, 2008; Mange et al., 2016), aunque se destacan los restos de especies acuáticas (Mange, 2019). En este trabajo se presentan los resultados del análisis de los restos óseos y las cáscaras de huevos de aves provenientes de cuatro cuadrículas del sitio Co1. Los objetivos son determinar cuáles fueron las causas o agentes responsables de la acumulación de los especímenes de aves y discutir el aprovechamiento de estos recursos en el sitio y su importancia en los valles fluviales de Norpatagonia en el Holoceno tardío.

\section{MATERIALES Y MÉTODOS}

El conjunto faunístico analizado $(n=1717)$ incluye restos óseos y cáscaras de huevos de aves recuperados en cuatro cuadrículas de un metro cuadrado cada una (C6, C7, C8 y C9), que fueron excavadas siguiendo niveles artificiales de $5 \mathrm{~cm}$ hasta los $50 \mathrm{~cm}$ de profundidad. Los sedimentos fueron tamizados con una malla metálica de 2,5 mm de diámetro. La determinación taxonómica y anatómica de los especímenes óseos se realizó mediante el empleo de muestras osteológicas comparativas de la Sección Ornitología, División Zoología de Vertebrados (FCNyM, UNLP). Mientras que los especímenes de grandes dimensiones fueron asignados sin dificultades a Rhea sp., los restos de especies menores que no pudieron determinarse fueron ubicados en categorías generales según su masa corporal: aves medianas (mayores a 200 g) y aves pequeñas (menores a 200 g). La determinación taxonómica de las cáscaras de huevos de aves medianas/pequeñas se realizó en base a características morfológicas (color, textura y grosor), utilizando muestras comparativas de la colección de aves previamente mencionada y bibliografía (De la Peña, 2019).

Para la cuantificación de los especímenes óseos se emplearon medidas de abundancia taxonómica (NISP, NMI) y anatómica (NME, MAU) siguiendo la propuesta de varios autores (Grayson, 1984; Lyman, 1994, entre otros). Las primeras fueron utilizadas para analizar la importancia relativa de cada especie, y las segundas para discutir la representación diferencial de partes anatómicas en el sitio. En la muestra de tinámidos se analizó el perfil anatómico con el objetivo de determinar si hubo supervivencia diferencial mediada por la densidad mineral ósea (Dirrigl, 2001) o algún tipo de manipulación antrópica diferencial de las partes (Laroulandie, 2005). Teniendo en cuenta que la densidad ósea en las aves está relacionada con los hábitos de comportamiento y locomoción (Cruz y Elkin, 2003; Laroulandie, 2005), se utilizaron los valores propuestos para Meleagris gallopavo, otra ave terrestre mediana (Dirrigl, 2001; Laroulandie, 2005). Por otro lado, el número mínimo de huevos de ñandú se calculó siguiendo a Quintana (2008).

Con el objetivo de conocer cuáles fueron los agentes de acumulación del conjunto y las alteraciones posdepositacionales, se relevaron los tipos de fractura y diversos efectos tafonómicos sobre la superficie cortical de los especímenes óseos: huellas antrópicas, termoalteración, marcas de carnívoros y roedores, corrosión digestiva, marcas de raíces, pisoteo y depositación de carbonato de calcio (Binford, 1981; Olsen y Shipman, 1988; Lyman, 1994; Bochenski y Tomek, 1997; Montalvo, 2002; Gutiérrez, 2004). En la muestra de cáscaras de huevos se relevó la presencia de termoalteración y de grabados o incisiones (Fiore y Borella, 2010). Todos los elementos fueron estudiados a ojo desnudo y en lupa binocular con aumento de hasta 20x.

\section{RESULTADOS}

La muestra analizada incluye cáscaras de huevos $(\mathrm{NISP}=1375, \mathrm{NMI}=8)$ y restos óseos $(\mathrm{NISP}=342$, 
$\mathrm{NMI}=18)$. Entre los especímenes óseos predominan los de aves medianas (NISP\% = 91,2), principalmente de tinámidos (Tabla 1), mientras que son escasos los restos de rheidos $(3,8 \%)$ y aves pequeñas (5\%). La especie más abundante es Eudromia elegans, tanto en especímenes (NISP\% $=33,6)$ como en individuos ( $\mathrm{NMI}=7$, obtenido en base a coracoides izquierdo). Otros restos de tinámidos corresponden a indeterminados (Tinamidae indet.) y a Nothura sp. Aunque los especímenes de otras aves de tamaño mediano son escasos, se determinaron elementos de estrigiformes (Strigiformes indet.), falcónidos (Falconidae indet.), y de dos grupos de aves acuáticas, patos (Anatidae) y biguás (Phalacrocorax sp.). Entre las cáscaras (Tabla 1) predominan especímenes de Rhea sp., con un peso de 251 g y correspondientes, al menos, a cuatro huevos. En menor cantidad se identificaron cáscaras de E. elegans, Nothura sp. y de aves medianas indeterminadas con distintas características: de color blanco crema con superficie rugosa $(n=14)$ y de color blanco con superficie lisa $(n=130)$, que a su vez presentan dos variantes: opacas y satinadas.

La representación anatómica de E. elegans muestra el predominio significativo de la porción proximal del coracoides, y también de la región proximal de escápula, el esternón y la epífisis distal de tibiotarso, con valores de MAU mayores al 50\% (Figura 2). En menor abundancia siguen la región distal de coracoides, el sinsacro, la hemipelvis y las epífisis distales de radio y ulna; otras unidades anatómicas tienen baja representación. La comparación entre las frecuencias de las unidades anatómicas y los valores de densidad mineral ósea de Meleagris gallopavo (Dirrigl, 2001) permite proponer que la representación anatómica no habría sido afectada por procesos atricionales mediados por la densidad ósea (Figura 3). Se hallaron elementos no fusionados de esta especie (dos fémures derechos y un tibiotarso), lo que indicaría también la captura de individuos juveniles $(\mathrm{NMI}=2)$. Aunque la muestra es considerablemente más pequeña, entre los restos de Nothura sp. se observa una tendencia similar (Figura $3)$, con predominio de elementos de menor densidad (sinsacro y coracoides). En los especímenes indeterminados de Tinamidae abundan los fragmentos del sinsacro (NISP $=8$ ), y en baja proporción se hallaron porciones distales de coracoides y proximales de tibiotarso y de esternón, es decir, también elementos con baja densidad (Dirrigl, 2001). Los elementos de otros grupos de aves medianas son diversos: mandíbula, metacarpiano, esternón (quilla), porción distal de coracoides y falange de anátidos, sinsacro y metacarpiano de Phalacrocorax sp., coracoides, tarsometatarso y falange de estrigiforme, y dos fragmentos distales de coracoides y una epífisis distal de húmero de falcónidos.

La muestra de rheidos está representada por varios elementos de los miembros posteriores (dos

\begin{tabular}{|c|c|c|c|c|c|c|c|c|}
\hline & TAXA & NV & NISP & NISP \% & TA & FF & HA & NMI \\
\hline \multirow{11}{*}{$\begin{array}{c}\text { Restos } \\
\text { óseos }\end{array}$} & Rhea sp. & Ñandúes & 13 & 3,8 & 4 & 2 & 2 & 2 \\
\hline & Ave indet. (med.) & Aves medianas & 130 & 38 & 56 & 26 & 8 & - \\
\hline & Strigiformes indet. & Estrigiformes & 4 & 1,2 & 0 & 0 & 0 & 1 \\
\hline & Falconidae indet. & Falcónidos & 3 & 0,9 & 2 & 3 & 0 & 1 \\
\hline & Phalacrocorax sp. & Biguás & 2 & 0,6 & 1 & 2 & 0 & 1 \\
\hline & Tinamidae indet. & Tinámidos & 39 & 11,4 & 19 & 7 & 0 & - \\
\hline & Eudomia elegans & Martineta & 115 & 33,6 & 40 & 39 & 18 & 7 \\
\hline & Nothura sp. & Perdices & 14 & 4,1 & 6 & 6 & 5 & 3 \\
\hline & Anatidae indet. & Anátidos & 5 & 1,5 & 2 & 2 & 0 & 1 \\
\hline & Ave indet. (peq.) & Aves pequeñas & 12 & 3,5 & 5 & 2 & 1 & - \\
\hline & Passeriformes indet. & Pájaros & 5 & 1,5 & 0 & 2 & 0 & 2 \\
\hline \multicolumn{3}{|r|}{ Subtotal } & 342 & 100 & 135 & 91 & 34 & 18 \\
\hline \multirow{4}{*}{ Cáscaras } & Rhea sp. & Ñandúes & 1194 & 86,8 & 349 & - & 15 & 4 \\
\hline & Ave indet. (med.) & Aves medianas & 144 & 10,5 & 9 & - & 0 & 2 \\
\hline & Nothura sp. & Perdices & 14 & 1,0 & 0 & - & 0 & 1 \\
\hline & Eudromia elegans & Martineta & 23 & 1,7 & 1 & - & 0 & 1 \\
\hline \multicolumn{3}{|r|}{ Subtotal } & 1375 & 100 & 359 & - & 15 & 8 \\
\hline \multicolumn{3}{|r|}{ TOTAL } & 1717 & - & 494 & 91 & 49 & 26 \\
\hline
\end{tabular}

Tabla 1. Representación taxonómica de los restos óseos y cáscaras de huevos analizados y los nombres vulgares (NV) utilizados. Se indican los especímenes termoalterados (TA), con fracturas en estado fresco (FF) y con huellas antrópicas (HA). epífisis distales de tibiotarso, una diáfisis y una epífisis proximal de tarsometatarso, cinco falanges 2 y una falange 3) y escasos elementos del esqueleto axial (dos vértebras) y de los miembros anteriores (una epífisis distal de radio). Se identificaron elementos con densidades variables (por ejemplo, cuerpo vertebral y diáfisis de tarsometatarso, véase Cruz y Elkin, 2003), lo 


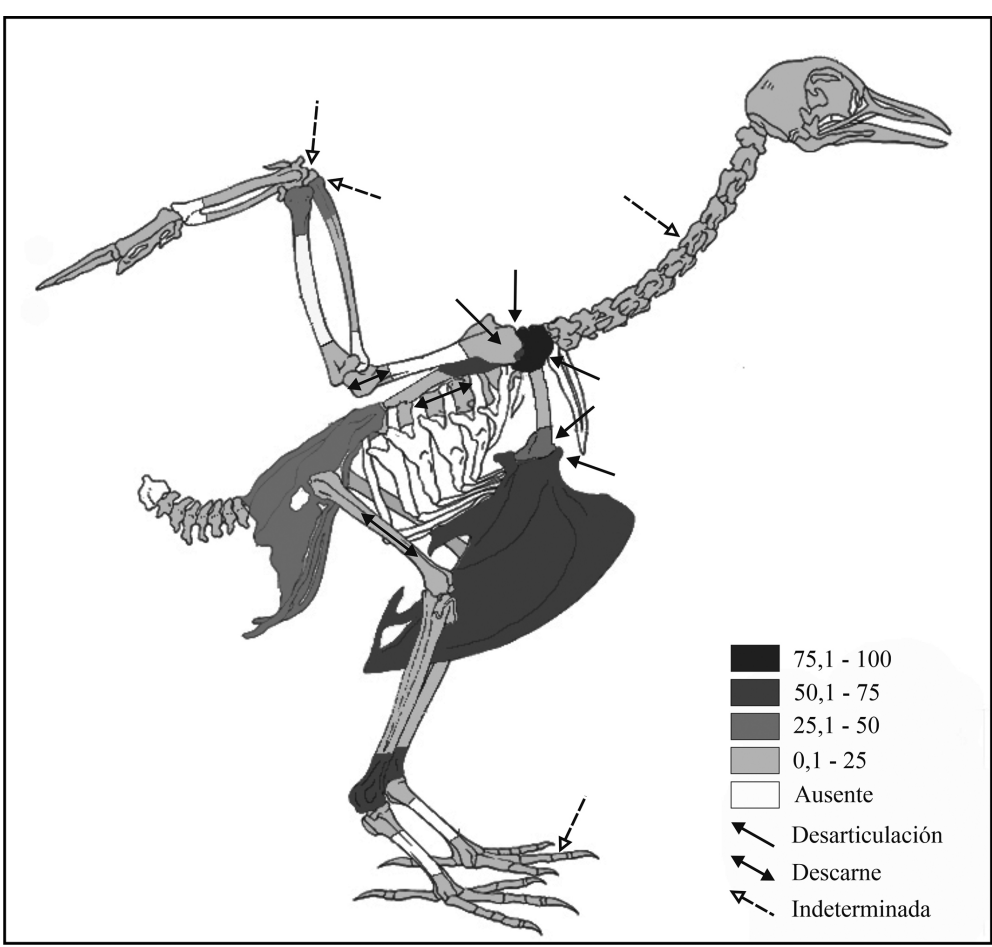

Figura 2. Representación de las partes esqueletarias (MAU\%) de Eudromia elegans y ubicación de las huellas de procesamiento.

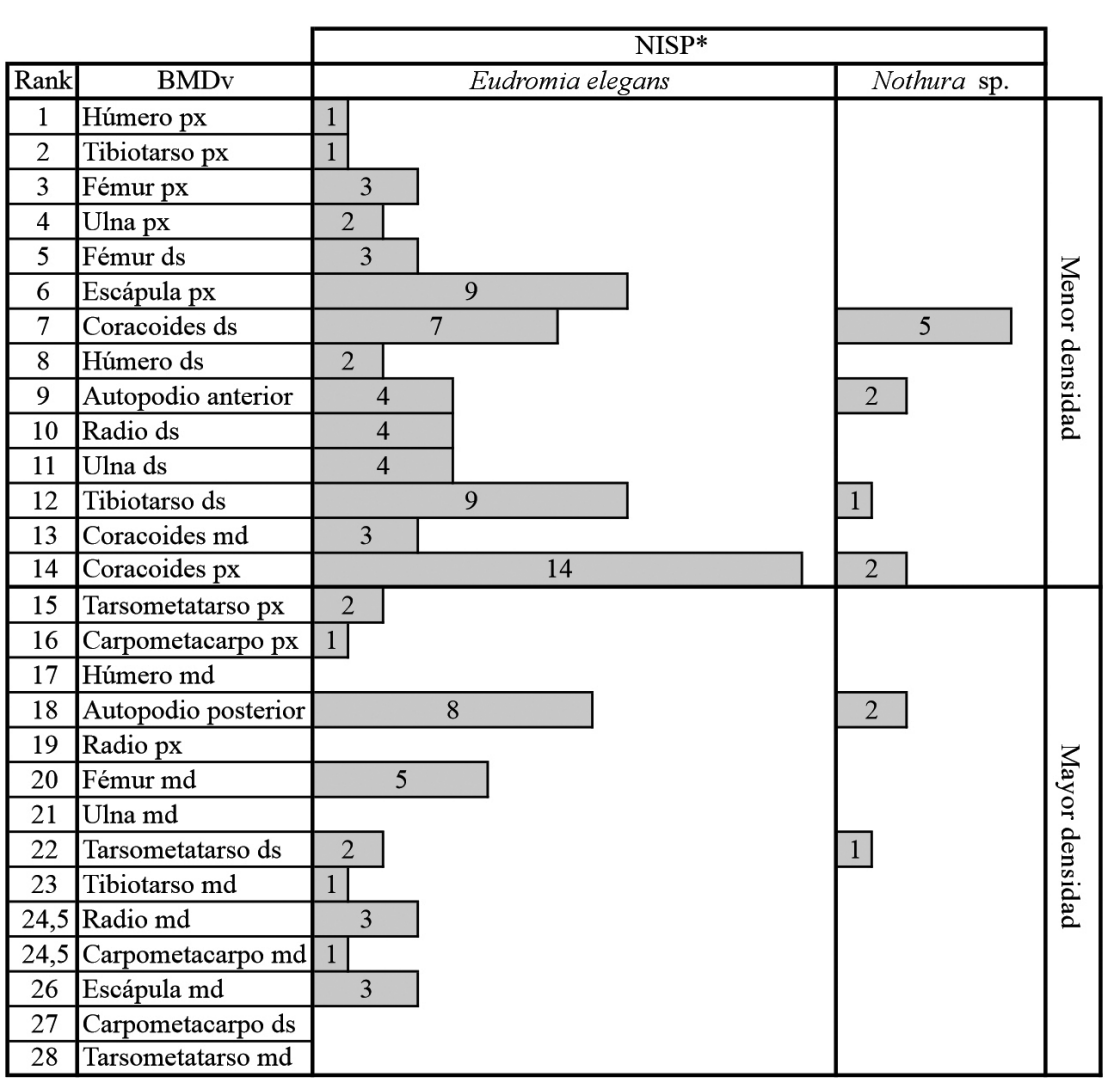

Figura 3. Frecuencia $\left(\mathrm{NISP}^{*}\right)$ de algunos elementos de E. elegans y Nothura sp. ranqueados de menor a mayor en función de los valores de densidad mineral ósea por volumen (BMDv; Dirrigl, 2001).

cual apoya la propuesta de que los procesos atricionales no habrían tenido una incidencia importante en la representación de las unidades anatómicas.
Se hallaron elementos no fusionados (una epífisis proximal de falange 2 y un cuerpo vertebral) y fusionados (tres falanges 2, un tarsometatarso y un radio), lo que indica que se habrían ingresado al sitio al menos un individuo adulto y otro juvenil.

El análisis tafonómico mostró una elevada incidencia de fragmentación en estado seco $(75,9 \%)$, siendo escasos los elementos enteros en la muestra analizada (12,3\%). En numerosos especímenes se observaron marcas de raíces $(36,9 \%)$ y depositaciones de carbonatos de calcio $(20,7 \%)$, y en algunos restos de aves medianas y pequeñas se detectó corrosión digestiva leve a moderada $(\mathrm{n}=10$; $3,6 \%)$. Además, la incidencia de raíces $(20-46 \%)$ y carbonatos (11$36 \%$ ) es similar en los especímenes de distintos taxones. Las evidencias de procesamiento antrópico sobre los especímenes óseos incluyen: huellas de corte y raspado (en el $10 \%$ de la muestra), termoalteración $(39,5 \%)$ y fracturas en estado fresco $(30,8 \%)$. Las huellas se registraron en 34 especímenes, principalmente de $E$. elegans (Figura 2), y también de aves medianas indeterminadas, Nothura sp., Rhea sp. y aves pequeñas (Tabla 2). En restos de martinetas y perdices (Figura 2, Tabla $2)$, se concentran en torno a dos porciones anatómicas: en la articulación entre el húmero y la cintura escapular y en la articulación entre el coracoides y el esternón (Figuras 4A-C), lo que indica la desarticulación de estas porciones anatómicas. Algunas huellas en restos de aves medianas indeterminadas (Tabla 2) se ubican también en cercanías de esas articulaciones. Sobre los especímenes de Rhea sp. se identificaron huellas de corte en una diáfisis de tarsometatarso y sobre una 


\begin{tabular}{|c|c|c|c|c|c|c|}
\hline & & Rhea sp. & $\begin{array}{l}\text { Aves } \\
\text { med. }\end{array}$ & E. elegans & $\begin{array}{l}\text { Nothura } \\
\text { sp. }\end{array}$ & $\begin{array}{l}\text { Aves } \\
\text { peq. }\end{array}$ \\
\hline Esternón & Craneal & - & 2 & 3 & - & - \\
\hline \multirow{2}{*}{ Coracoides } & Proximal & - & - & 3 & 1 & - \\
\hline & Distal & - & - & 1 & 2 & - \\
\hline Escápula & Proximal & - & - & 2 & - & - \\
\hline \multirow{3}{*}{ Húmero } & Proximal & - & - & 1 & - & - \\
\hline & Diáfisis & - & 1 & - & - & - \\
\hline & Distal & - & - & 1 & - & - \\
\hline Radio & Distal & - & - & 2 & - & - \\
\hline Metacarpiano & Proximal & - & - & 1 & - & - \\
\hline Fémur & Diáfisis & - & - & 1 & - & - \\
\hline Tibiotarso & Diáfisis & - & 1 & - & - & - \\
\hline Peroné & Proximal & - & 1 & - & - & - \\
\hline \multirow{3}{*}{ Tarsometatarso } & Proximal & - & - & - & 1 & 1 \\
\hline & Diáfisis & 1 & - & - & - & - \\
\hline & Distal & - & 1 & - & - & - \\
\hline \multicolumn{2}{|l|}{ Hemipelvis } & - & 1 & - & - & - \\
\hline \multicolumn{2}{|l|}{ Autopodio } & 1 & 1 & 1 & 1 & - \\
\hline \multicolumn{2}{|l|}{ Vértebras } & - & - & 2 & - & - \\
\hline \multicolumn{2}{|l|}{ TOTAL } & 2 & 8 & 18 & 5 & 1 \\
\hline
\end{tabular}

Tabla 2. Elementos óseos con huellas de corte y/o raspado.

aves pequeñas, anátidos, falcónidos, biguá y varios de ñandú (vértebra cervical, tibiotarso y dos falanges). En las cáscaras de huevos se registró termoalteración en especímenes de rheidos $(29,2 \%)$, de aves medianas indeterminadas $(6,3 \%)$ y de $E$. elegans $(4,3 \%)$.

Las fracturas producidas en estado fresco se registraron en especímenes de la mayoría de los taxa, con excepción de estrigiformes (Tabla 1). En falcónidos y biguás, ambos con escasa representación, todos los especímenes

falange 2. Además, se registraron huellas de tipo grabado en algunas cáscaras de huevos de rheidos ( $n=15 ; 1,3 \%$; Figura $4 D$ ), aunque no se identificaron motivos debido al tamaño de los fragmentos.

Las evidencias de alteración térmica se registraron en especímenes de la mayoría de los grupos taxonómicos, con excepción de estrigiformes y passeriformes (Tabla 1). En los restos de tinámidos la termoalteración es importante, tanto en indeterminados $(48,7 \%)$, como en los de perdices $(42,8 \%)$ y martinetas $(34,8 \%)$. También la incidencia es elevada en especímenes de aves medianas indeterminadas $(43,1 \%)$. En todos los casos afecta a elementos del esqueleto axial y apendicular, excepto en perdices, donde solo se presenta en coracoides, tibiotarso y falange. En E. elegans se observó alteración térmica en elementos del esqueleto axial (ocho vértebras y dos extremos craneales de esternón) y apendicular: porciones proximales $(\mathrm{n}=4)$ y distales de coracoides $(\mathrm{n}=5$; Figura $4 \mathrm{C})$, proximales de escápula $(\mathrm{n}=5)$, epífisis proximales de húmero, ulna y fíbula, hemipelvis, epífisis proximal y dos porciones de diáfisis de fémur, dos epífisis distales de tibiotarso y tarsometatarso, dos proximales de tarsometatarso y cinco falanges. También se hallaron especímenes termoalterados de

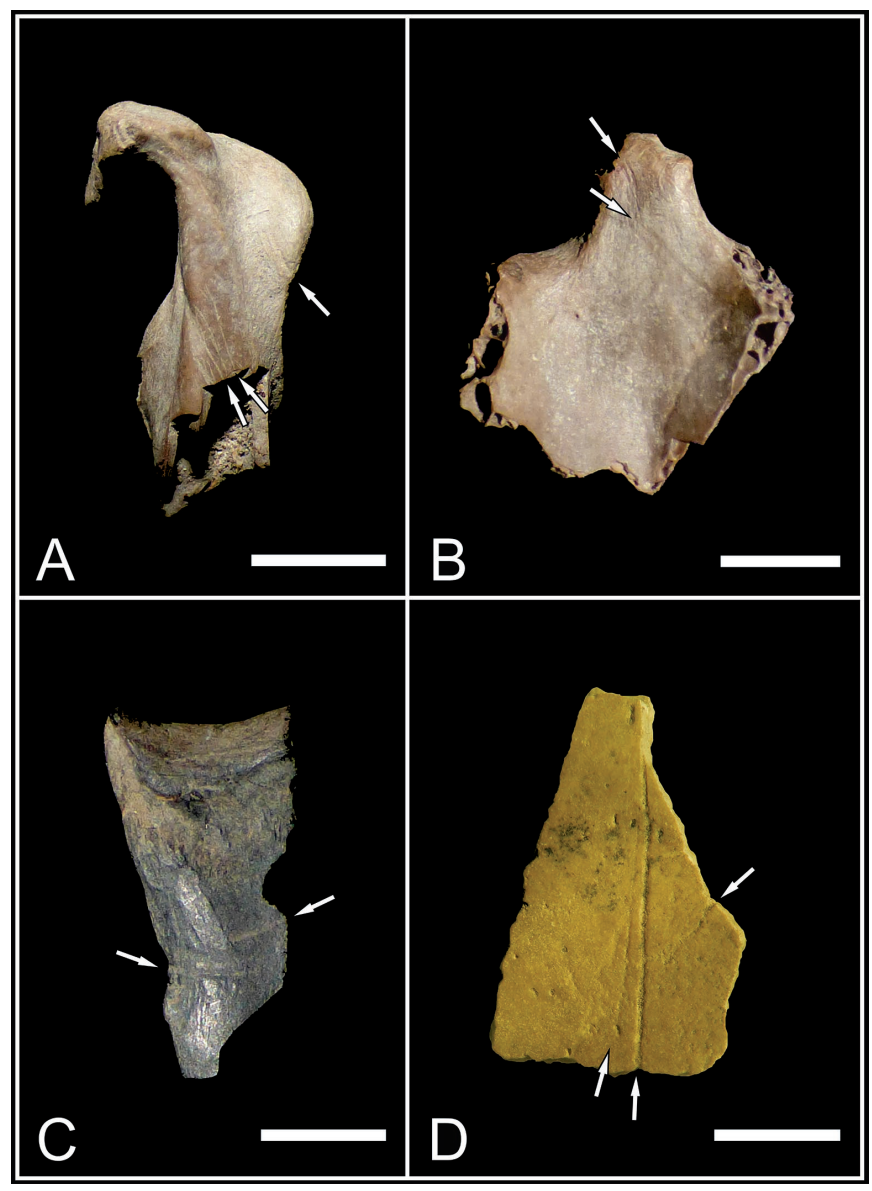

Figura 4. Evidencias de procesamiento antrópico: porciones proximal (A) y distal (C) de coracoides y extremo craneal de esternón (B) de $E$. elegans con huellas de corte y termoalteración; D) grabado en cáscara de huevo de Rhea sp. Escalas $=5 \mathrm{~mm}$. 
presentan fractura en fresco; también su incidencia es elevada en la muestra reducida de anátidos (40\%). Asimismo, este efecto es importante en los restos de tinámidos: en martineta $(33,9 \%)$ se observó en coracoides ( $n=10)$, escápulas $(n=6)$ y numerosas diáfisis de huesos largos ( $\mathrm{n}=19)$; en perdices $(42,6 \%)$, en coracoides $(n=5)$ y un tibiotarso; y en tinámidos indeterminados $(17,9 \%)$, en huesos largos $(n=4)$, vértebras $(n=2)$ y esternón. En la muestra de aves medianas se registraron fracturas en estado fresco en el $20 \%$ de los especímenes y también en tarsometatarso y radio de Rhea sp.

\section{DISCUSIÓN}

El conjunto faunístico analizado en este trabajo incluye restos óseos (nTaxa $=8)$, mayormente de aves medianas $y$, en menor medida, de ñandúes $y$ aves pequeñas, y fragmentos de cáscaras de huevos (nTaxa $=6$ ). Los resultados del análisis permiten proponer que el conjunto es principalmente de origen antrópico, considerando que el 58\% de los restos óseos y el $27 \%$ de las cáscaras presentan evidencias de procesamiento (e.g., termoalteración, fracturas en estado fresco o huellas), y también que los especímenes se hallaron dispersos en la matriz sedimentaria y estrechamente asociados a diversos artefactos (Mange, 2019). El perfil taxonómico de los materiales óseos evidencia el predominio de aves medianas (más del 90\% de la muestra) y en particular de tinámidos (ca. 50\% del total). Otras aves medianas que se habrían consumido son anátidos, biguás y falcónidos, de acuerdo con la termoalteración y las fracturas en estado fresco que presentan los escasos elementos. Los ñandúes, las aves de mayor rendimiento cárnico disponibles, se encuentran escasamente representados aunque presentan evidencias de procesamiento y el consumo de sus huevos habría sido importante. De manera similar, en la reducida muestra de restos de aves pequeñas también se hallaron evidencias de modificación antrópica.

En forma secundaria, de acuerdo con la presencia de corrosión leve en algunos especímenes, puede proponerse la acción de depredadores, probablemente aves estrigiformes, como agentes de acumulación del conjunto. Se planteó previamente, en base a observaciones actualísticas realizadas en el valle del río Negro, que Athene cunicularia, que puede habitar en cuevas sobre los mismos médanos, puede incorporar restos óseos a los sitios arqueológicos (Mange, 2019). Estas estrigiformes podrían ser responsables del ingreso de especímenes de aves pequeñas (De Tommaso et al., 2009; Montalvo et al., 2020) y haber causado las fracturas en estado fresco que presentan (Rufà et al., 2015). Los especímenes de estrigiformes de la muestra no poseen ningún tipo de modificación relacionada con el aprovechamiento antrópico, tal como fue observado en sitios cercanos (Prates, 2008; Mange, 2019), y podrían haber ingresado por causas naturales. Aunque las aves medianas y especialmente los tinámidos forman parte de la dieta de carnívoros como los zorros (Fernández et al., 2010; De la Peña 2019), estos producen modificaciones que no fueron observadas en la muestra (e.g., perforaciones, corrosión fuerte, etc.) (Mondini, 2002; Fernández et al., 2010). Además, los sectores abiertos del interior del valle pueden constituir espacios de caza, pero no son lugares donde se depositan restos óseos en madrigueras o letrinas (Mondini, 2002). Por último, puede mencionarse que algunas aves rapaces diurnas presentes en la zona (e.g., Falco spp. y Circus spp.) consumen diversas aves, incluyendo tinámidos, aunque difícilmente hayan usado los médanos como posaderos, por lo que se descarta su rol como agentes acumuladores (véanse Narosky y Babarskas, 2001; Bó et al., 2007).

De acuerdo con el perfil taxonómico, es probable que muchos de los especímenes óseos de aves medianas no determinadas -algunos con evidencias de modificación antrópica- correspondan a alguna de las especies de tinámidos que habitan Norpatagonia centro-oriental (Narosky y Babarskas, 2001): Rhynchotus rufescens, Nothoprocta cinerascens, y más probablemente a Eudromia elegans, Nothura maculosa y Nothura darwinii. Teniendo en cuenta que los tinámidos habitan estepas arbustivas y pastizales (Narosky y Babarskas, 2001), su captura podría haber ocurrido en el interior del valle, cerca de los campamentos. Las martinetas -la especie más explotada en Co1- se caracterizan por ser aves corredoras con poca capacidad de vuelo, hábitos sociales y un peso superior a los 500 g (De la Peña, 2019), y habrían sido un recurso predecible y de buen rendimiento. El aprovechamiento de tinámidos ha sido mencionado para otros sitios del valle del río Negro (Prates y Acosta Hospitaleche, 2010; Mange, 2019), así como en la costa del golfo San Matías (Marani y Borella, 2014), el curso inferior del río Colorado (Alcaráz, 2017) y el valle medio/ inferior del río Limay (Tonni, 1981). El análisis de las huellas de corte en los elementos de tinámidos 
y aves medianas de Co1 permitió establecer algunas pautas de manipulación de las carcasas que indican su procesamiento, cocción y consumo en el sitio (cf., Laroulandie, 2001, 2005):

a) las huellas situadas en la epífisis proximal del húmero (uno de martineta) y sobre las porciones proximales del coracoides (tres de martineta y uno de perdiz) y de la escápula (dos de martinetas) podrían relacionarse con el seccionamiento del ala como una unidad;

b) aquellas ubicadas en la porción distal del coracoides (uno de martineta y dos de perdiz) y en la región craneal del esternón (tres de martineta, dos de ave mediana) indicarían la obtención de los músculos pectorales y supracoracoideos;

c) las ubicadas en cercanías de las epífisis distales de tarsometatarso (uno de ave mediana y uno de perdiz), en las falanges (de martineta, perdiz y ave mediana) y en epífisis distales de radio (dos de martineta) y metacarpiano (martineta) señalarían el seccionamiento y descarte de los extremos de miembros anteriores y posteriores;

d) las huellas en la epífisis proximal de la fíbula (de ave mediana) y en epífisis distal de húmero (de martineta) podrían relacionarse con el trozamiento y reparto previos al consumo;

e) las escasas huellas de corte en diáfisis de huesos largos (fémur, tibiotarso y húmero) estarían asociadas con el descarne previo o más probablemente durante el consumo.

Con excepción de un tarsometatarso de estrigiforme, todos los huesos largos de aves medianas del sitio se encuentran fracturados (el 59\% en estado fresco), lo cual se relaciona con el trozamiento de las carcasas (Laroulandie, 2005). Complementariamente, las evidencias de termoalteración en sectores articulares de elementos de $E$. elegans permiten proponer un patrón vinculado a las unidades anatómicas expuestas al fuego (Laroulandie, 2001, 2005). El quemado de las porciones proximales de escápula, coracoides y húmeros indicaría la cocción de las carcasas y las alas luego de su disección. De manera similar, la termoalteración de las epífisis proximal de fémur y distal de tibiotarso podría indicar el asado de los miembros posteriores como otra unidad. Por último, algunos especímenes presentan ese patrón de termoalteración y huellas de corte vinculadas al descarne $(\mathrm{n}=3)$, lo que indicaría que esta última actividad se realizó luego de la cocción mediante asado (Cassoli y Tagliacozzo, 1997). Debe tenerse en cuenta que la cocción previa de las presas permite un mejor desprendimiento de la masa muscular y que el procesamiento de presas pequeñas no siempre deja evidencia de manipulación en los huesos (Blasco et al., 2014).

Otras aves medianas consumidas en Co1 habrían sido anátidos y biguás, comunes en ríos y lagos norpatagónicos y especialmente en ambientes eutróficos como lagunas y cauces semiactivos (Narosky y Babarskas, 2001). Estas aves habrían sido capturadas en el cauce ubicado en cercanías del campamento, donde se habrían obtenido otros recursos acuáticos, como peces y moluscos (Mange, 2019). Las aves acuáticas han sido identificadas en los conjuntos de otros sitios del valle medio del río Negro (Prates y Acosta Hospitaleche, 2010; Mange, 2019). La muestra más diversa proviene del sitio Angostura 1, donde se registraron restos de Anas sp. termoalterados y con una huella de corte, de Fulica sp. con termoalteración y elementos no modificados de Himantopus sp. y Podiceps major (Prates y Acosta Hospitaleche, 2010). En los sectores inferiores de las tres grandes cuencas de Norpatagonia (ríos Colorado, Negro y Chubut), las sociedades cazadoras-recolectoras del Holoceno tardío habrían hecho un uso sostenido (sensu Prates y Bonomo, 2017) de los ambientes acuáticos y sus recursos (Prates y Acosta Hospitaleche, 2010; Alcaráz, 2015, 2017; Svoboda y Gómez Otero, 2015; Mange, 2019).

Aunque los restos óseos de ñandúes son escasos, presentan evidencias claras de aprovechamiento antrópico y habrían sido una fuente importante de alimento considerando su peso (hasta $30 \mathrm{~kg}$ para Rhea americana y $24 \mathrm{~kg}$ para $R$. pennata) (De la Peña, 2019). Los ñandúes habitan estepas arbustivas y pastizales, aunque el valle del río Negro habría actuado como un atractor por la presencia de pastizales, agua y sedimentos blandos para nidificar (véanse D'Orbigny, 1945, p. 790; Tambussi, 1995). De esta manera, probablemente fueron capturados en el interior del valle, que presenta rasgos que permiten optimizar el avistaje e intercepción de presas veloces (véase discusión en Mange, 2019). La presencia de elementos axiales y de ambos miembros indica el ingreso de carcasas enteras al sitio, y las huellas observadas podrían relacionarse con el descarnado de huesos largos (tarsometatarso) y la extracción de tendones (falange). La escasez de especímenes en Co1 y en otros sitios arqueológicos prehispánicos del valle medio del río Negro (Prates, 
2008; Mange et al., 2016; Mange, 2019) contrasta con las numerosas referencias históricas que consideraron a estas aves como el alimento preferido por los pueblos indígenas norpatagónicos (Casamiquela, 1985; Salemme y Frontini, 2011). Una situación similar ha sido observada en zonas cercanas, como el golfo San Matías (Marani, 2016), el valle inferior del río Colorado (Stoessel y Alcaráz, 2017) y el sector medio/superior del río Limay (Cordero, 2010). Una explicación a esta disparidad podría estar relacionada con los cambios sociales ocurridos desde el siglo XVI en las regiones pampeano-patagónicas y con las características propias de estas aves, que son excelentes corredoras y, por lo tanto, podrían haber sido presas difíciles de capturar en el período preecuestre (Giardina, 2010; Salemme y Frontini, 2011). La introducción del caballo en Norpatagonia durante los siglos XVI-XVII (Fernández, 2006) permitió una mayor velocidad y modificó las estrategias de caza, e incluso el transporte y consumo de sus partes.

Por el contrario, los huevos de ñandú habrían sido un componente importante de la dieta de las poblaciones prehispánicas que ocuparon este sitio y otros de los valles norpatagónicos (Prates y Acosta Hospitaleche, 2010; Martínez, 2015; Fernández et al., 2016; Mange, 2019) y de la costa del golfo San Matías (Marani, 2016). Las cáscaras termoalteradas recuperadas indican que los huevos habrían sido cocinados al rescoldo, tal como fue observado por cronistas y viajeros (e.g., Musters, 1997). Por otro lado, los grabados sobre algunos de los fragmentos de cáscaras recuperados en Co1 y en diversos sitios de Norpatagonia dan cuenta del uso más allá del consumo alimenticio, posiblemente como contenedores (Fiore y Borella, 2010; Carden y Martínez, 2014). Además, en Co1 se habrían aprovechado huevos de algunas aves medianas (E. elegans, Nothura sp. y otras). Aunque los tinámidos hacen sus nidos en sustratos blandos, y en particular $E$. elegans podría anidar sobre los mismos médanos (De la Peña, 2019), las cáscaras de sus huevos son frecuentes en sitios del valle medio del río Negro (Prates y Acosta Hospitaleche, 2010; Mange, 2019), y puede considerarse probable su ingreso antrópico. Otros tinámidos presentes en el área prefieren nidificar en pastizales (De la Peña, 2019) y por lo tanto, sería menos probable el ingreso natural de las cáscaras de huevo. Además, los cauces y lagunas son sectores donde anidan varias especies (Anas spp., Fulica spp., Chloephaga sp. y otras; véase Narosky y Babarskas, 2001), cuyos huevos habrían estado disponibles en cercanías del campamento en primavera y verano (Musters, 1997, p. 142). Es importante agregar que en Co1 se hallaron materiales que indican la presencia humana en época estival (e.g., endocarpos de Condalia microphylla) (Mange, 2019).

El análisis arqueofaunístico de Co1 es preliminar y hasta el momento se han estudiado solo los materiales de una cuadrícula, aunque con una gran cantidad de especímenes faunísticos óseos (NSP = 6208) y valvas de moluscos (NISP = 787) (Mange, 2019). Esos resultados mostraron que, al igual que en otros sitios del valle medio del río Negro (Prates, 2008; Mange et al., 2016), el consumo faunístico habría estado basado en la explotación de artiodáctilos (Lama guanicoe y Ozotoceros bezoarticus; NISP\% = 8,5), armadillos (Chaetophractus villosus y Zaedyus pichiy, NISP\% = 22,9), roedores (Microcavia australis, Galea leucoblephara y Holochilus vulpinus, NISP\% = 25,8), moluscos (Diplodon chilensis) y, en menor medida, aves (NISP\% $=4 \%) ; y$ por otro lado, destacaron la elevada cantidad de restos de peces (Percichthys trucha y Odontesthes sp.; NISP\% $=5,5)$. Los nuevos análisis realizados con las muestras de las cuadrículas 6-9, incluyendo los presentados en este trabajo, permiten agregar a las aves como un componente más importante en la dieta de los cazadores-recolectores del Holoceno tardío. La comparación entre los especímenes de artiodáctilos y aves (Índice de aves = NISP de aves/ NISP de aves + NISP de artiodáctilos) entre Co1 y los otros sitios de la zona excavados sistemáticamente (Negro Muerto, Pomona y Angostura 1) (Prates, 2008; Mange, 2019; Mange et al., 2020) muestra valores más altos $y$, por lo tanto, una mayor importancia de las aves, en $\operatorname{NM}(0,6)$ y Co1 $(0,55)$, con respecto a $\mathrm{Po}(0,14)$ y $\mathrm{A} 1(0,36)$.

Los resultados del análisis tafonómico mostraron también una importante fragmentación del conjunto y la incidencia de raíces y carbonato, cuyos efectos son frecuentes en conjuntos arqueofaunísticos del valle medio del río Negro (Mange et al., 2016; Mange et al., 2020). Esta elevada fragmentación, ocurrida fundamentalmente cuando los huesos estaban en estado seco, se relacionaría con la fragilidad intrínseca de los huesos de aves (Dirrigl et al., 2020), con la abundante incidencia de termoalteración, con la actividad radicular de los arbustos que cubren este sector del valle y con la acción de animales fosoriales (roedores y eufractinos). La presencia de marcas de raíces, el predominio de estadios bajos o ausencia de meteorización en la muestra de mamíferos y la escasez de especímenes 
con blanqueamiento (Mange, 2019) indican que la movilidad de los sedimentos del médano no habría sido tan importante, al menos en el sector excavado, donde habrían predominado las condiciones de enterramiento desde la ocupación humana, hace aproximadamente 700 años.

\section{CONSIDERACIONES FINALES}

La evidencia presentada en este trabajo muestra que las aves constituyeron un componente frecuente de la dieta de los grupos cazadores-recolectores del valle del río Negro. Esto no había sido señalado previamente para los sitios arqueológicos de esta zona. El aprovechamiento antrópico estuvo enfocado en los tinámidos (especialmente en martinetas) como recurso cárnico, y en los huevos de ñandú, aunque se explotaron diversas aves para el consumo de carne y huevos. En los tinámidos, se observó un énfasis en el trozamiento y aprovechamiento de la región pectoral, la unidad anatómica con mayor rendimiento cárnico en estas aves y en otras de porte y hábitos afines (Laroulandie, 2005; Marani y Borella, 2014). Un procesamiento similar en carcasas de martinetas y aves acuáticas fue registrado en otros sitios del valle del río Negro y regiones vecinas (Prates y Acosta Hospitaleche, 2010; Marani y Borella, 2014; Alcaráz, 2015). También pueden resaltarse los hallazgos en Co1 de restos óseos de falcónidos (Falconidae indet.) y biguás (Phalacrocorax sp.), que tendrían orígen antrópico y son novedosos para la zona. Las cáscaras de huevo de ñandúes y tinámidos indican la ocupación del sitio en primavera/verano, aunque, como fue mencionado, esto no invalida ocupaciones en otros momentos del año, continuas o no.

Las huellas en elementos óseos de aves pequeñas, ya observadas en conjuntos faunísticos del valle medio del río Negro (Prates y Acosta Hospitaleche, 2010), muestran la diversidad de recursos explotados por las poblaciones cazadoras-recolectoras prehispánicas. Durante el Holoceno tardío se habrían consumido grandes vertebrados (guanacos, venados y ñandúes), aunque la dieta habría estado basada en recursos más pequeños y predecibles, tanto acuáticos (almejas, peces, rata-nutrias) como terrestres, obtenidos en zonas bajas del valle (eufractinos, cuises y diversos frutos) (Prates, 2008; Capparelli y Prates, 2015; Mange et al., 2016; Mange, 2019). Aunque el consumo importante de presas de menor rendimiento podría deberse a procesos de intensificación, tal como fue propuesto para zonas vecinas (e.g., Stoessel y Martínez, 2014), la falta de conjuntos faunísticos más antiguos en el valle medio del río Negro no permite contrastar esta hipótesis. Los análisis aquí presentados dan fuerza al modelo de aprovechamiento de diversos alimentos y permiten considerar que las aves medianas fueron recursos importantes obtenidos en zonas fluviales (e.g., anátidos y biguás) y en el interior del valle (principalmente tinámidos, incluyendo sus huevos). Para los valles de otros ríos norpatagónicos se han propuesto modelos de subsistencia similares para el Holoceno tardío (Cordero, 2010; Crivelli Montero, 2010; Svoboda y Gómez Otero, 2015; Fernández et al., 2016; Stoessel y Alcaráz, 2017), lo cual sería típico de poblaciones cazadoras-recolectoras en grandes valles fluviales, por la diversidad intrínseca de recursos que poseen estos ambientes (Ceruti y González, 2007).

\section{Agradecimientos}

Los trabajos fueron realizados en el marco de los proyectos PIP 244/15 y PICT 04547, dirigidos por Luciano Prates. Agradecemos también a Federico Scartascini y Matías Medina por la lectura crítica del trabajo, y a los/as evaluadores que con sus recomendaciones mejoraron sustancialmente el escrito.

\section{REFERENCIAS CITADAS}

Alcaráz, A. P. (2015). Aprovechamiento antrópico de fauna menor en el curso inferior del río Colorado (transición pampeano-patagónica oriental): el sitio El Tigre como caso de estudio. Intersecciones en Antropología, 16(1), 115-129.

Alcaráz, A. P. (2017). La fauna menor de sitios arqueológicos del curso inferior del río Colorado (provincia de Buenos Aires): aspectos tafonómicos y subsistencia de cazadores-recolectores durante el Holoceno medio y tardío [tesis doctoral inédita. Universidad Nacional del Centro de la provincia de Buenos Aires].

Binford, L. (1981). Bones. Ancient Men and Modern Myths. Academic Press.

Blasco, R., Finlayson, C., Rosell, J., Sánchez, A., Finlayson, S., Finlayson, G.,... y Rodríguez Vidal, J. (2014). The Earliest Pigeon Fanciers. Scientific Reports, 4(5971), 1-7.

Bó, M., Baladrón, A. y Biondi, L. (2007). Ecología trófica de Falconiformes y Strigiformes: tiempo de síntesis. El Hornero, 22(2), 97-115. 
Bochenski, Z. y Tomek, T. (1997). Preservation of bird bones: erosion versus digestion by owls. International Journal of Osteoarchaeology, 7, 372-387.

Bond, M., Caviglia, S. y Borrero, L. A. (1981). Paleoetnozoología del Alero de los Sauces (Neuquén, Argentina); con especial referencia a la problemática presentada por los roedores en sitios patagónicos. Trabajos de Prehistoria, 1, 95-111.

Borella, F., Cardillo, M., Favier Dubois, C. M. y Alberti, J. (2015). Nuevas investigaciones arqueológicas entre Punta Pórfido y Punta Odriozola: implicancias para el entendimiento de la dinámica de las ocupaciones humanas en la costa oeste del golfo San Matías (Río Negro). Relaciones de la Sociedad Argentina de Antropología, 40(1), 233-252.

Capparelli, A. y Prates, L. (2015). Explotación de frutos de algarrobo (Prosopis spp.) por grupos cazadoresrecolectores del Noreste de Patagonia. Chungara, 47(4), 549-563.

Carden, N. y Martínez, G. (2014). Diseños fragmentados: circulación social de imágenes sobre huevos de Rheidae en Pampa y Norpatagonia. Boletín del Museo Chileno de Arte Precolombino, 19(2), 55-75.

Casamiquela, R. M. (1985). Bosquejo de una etnología de la provincia de Río Negro. Fundación Ameghino.

Cassoli, P. y Tagliacozzo, A. (1997). Butchering and Cooking of Birds in the Palaeolithic Site of Grotta Romanelli (Italy). International Journal of Osteoarchaeology, 7, 303-320.

Ceruti, C. y González, M. I. (2007). Modos de vida vinculados con ambientes acuáticos del Nordeste y Pampa bonaerense de Argentina. Relaciones de la Sociedad Argentina de Antropología, 32, 101-140.

Cordero, J. A. (2010). Explotación animal en el Holoceno del noroeste de la Patagonia argentina. Cambios climáticos y transformaciones del comportamiento humano: una primera aproximación [tesis doctoral inédita. Universidad de Buenos Aires].

Crivelli Montero, E. (2010). Arqueología de la cuenca del río Limay. En R. Massera (Coord.), Los ríos mesetarios norpatagónicos. Aguas generosas del Ande al Atlántico (pp. 261-338). Gobierno de Río Negro.

Cruz, I. y Elkin, D. (2003). Structural bone density of the Lesser Rhea (Pterocnemia pennata) (Aves: Rheidae). Taphonomic and archaeological implications. Journal of Archaeological Science, 30(1), 37-44.

Cruz, I., Muñoz, S. y Zangrando, A. F. (2007). La interpretación de los restos de animales pequeños en la arqueología patagónica: Estado de la cuestión y perspectivas. En F. Morello, M. Martinic, A. Prieto y
G. Bahamonde (Eds.), Arqueología de Fuego-Patagonia. Levantando piedras, desenterrando huesos... y develando arcanos (pp. 15-22). Centro de Estudios del Cuaternario de Fuego Patagonia y Antártica.

De la Peña, M. (2019). Aves Argentinas: Descripción, comportamiento, reproducción y distribución (actualización). Tomo 1. Comunicaciones del Museo Provincial de Ciencias Naturales "Florentino Ameghino" (Nueva Serie), 1, 1-294.

De Tommaso, D., Callicó Fortunato, R., Teta, P. y Pereira, J. (2009). Dieta de la Lechucita Vizcachera (Athene cunicularia) en dos áreas con diferente uso de la tierra en el centro-sur de La Pampa, Argentina. El Hornero, 24(2), 87-93.

Dirrigl, F. (2001). Bone Mineral Density of Wild Turkey (Meleagris gallopavo) Skeletal Elements and its Effect on Differential Survivorship. Journal of Archaeological Science, 28, 817-832.

Dirrigl, F., Brush, T., Morales-Muñiz, A. y Bartosiewicz L. (2020). Prehistoric and historical insights in avian zooarchaeology, taphonomy and ancient bird use. Journal of Archaeological and Anthropological Sciences, 12(2), 57.

D’Orbigny, A. (1945 [1828-29]). Viaje por América Meridional. Tomo II. Futuro.

Favier Dubois, C. M., Kokot, R., Scartascini, F. y Borella, F. (2016). Una perspectiva geoarqueológica del registro de ocupaciones humanas en el Golfo San Matías (Río Negro, Argentina). Intersecciones en Antropología, 17(2), 47-59.

Fernández, F. J., Del Papa, L., Mange, E., Teta, P., Crivelli Montero, E. y Pardiñas, U. (2016). Human subsistence and environmental stability during the last 2200 years in Epullán Chica cave (Northwestern Patagonia, Argentina): a perspective from the zooarchaeological record. Quaternary International, 391, 38-50.

Fernández, F. J., Teta, P. y Pardiñas, U. (2017). Evidencias arqueológicas de explotación antrópica de micromamíferos en el extremo austral de América del Sur. Anales de Arqueología y Etnología, 72(1), 9-32.

Fernández, M. (2006). Economía y sistemas de asentamiento aborigen en la cuenca del río Limay. Memoria Americana, 14, 37-73.

Fernández, P., Cruz, I. y Forlano, A. (2010). Sitio 37: una madriguera de carnívoro en el norte de la Patagonia Andina (Provincia de Chubut, Argentina). En M. Gutiérrez, M. De Nigris, P. Fernández, M. Giardina, A. Gil, A. Izeta, G. Neme y H. Yacobaccio (Eds.), Zooarqueología a principios del siglo XXI, Aportes teóricos, metodológicos y casos de estudio (pp. 409417). Ediciones del Espinillo. 
Fiore, D. y Borella, F. (2010). Geometrías delicadas: Diseños grabados en cáscaras de huevo de Rheidae recuperados en la costa norte del Golfo San Matías, Río Negro. Intersecciones en Antropología, 11(2), 277-293.

Giardina, M. (2010). El aprovechamiento de la avifauna entre las sociedades cazadoras-recolectoras del sur de Mendoza [tesis doctoral inédita. Universidad Nacional de La Plata].

Grayson, D. (1984). Quantitative Zooarchaeology: topics in the analysis of archaeological faunas. Academic Press.

Gutiérrez, M. A. (2004). Análisis tafonómicos en el área Interserrana (provincia de Buenos Aires) [tesis doctoral inédita. Universidad Nacional de La Plata].

Laroulandie, V. (2001). Les traces liees a la boucherie, a la cuisson et a la consommation d'oiseaux: apport de I'experimentation. En L. Bourguignon, I. Ortega, M. Frere-Sautot (Eds.), Préhistoire et approche expérimentale (pp. 97-108). Monique Mergoual.

Laroulandie, V. (2005). Bird exploitation pattern: the case of Ptarmigan Lagopus sp. in the Upper Magdalenian site of La Vache (Ariège, France). En G. Grupe y J. Peters (Eds.), Feathers, grit and symbolism. Birds and humans in the ancient Old and New Worlds (pp. 165178). Verlag Leidorf.

Lyman, R. L. (1994). Vertebrate Taphonomy. Cambridge University Press.

Mange, E. (2019). Investigaciones arqueológicas en la margen sur del valle medio-superior del río Negro (provincia de Río Negro) [tesis doctoral inédita. Universidad Nacional de La Plata].

Mange, E., Fernández, F. J. y Di Lorenzo, M. (2020). Análisis del conjunto faunístico del sitio arqueológico Pomona (provincia de Río Negro, Argentina). Comechingonia, 24(3), 255-280.

Mange, E., Prates, L., González Venanzi, L. y Di Lorenzo, M. (2016). El registro faunístico del sitio Negro Muerto 3 (provincia de Río Negro, Argentina): tafonomía y patrones de explotación. Comechingonia, 20(1), 231-252.

Marani, H. (2016). Aprovechamiento de vertebrados terrestres por las poblaciones humanas que habitaron la costa del Golfo San Matías (Río Negro, Argentina) durante el Holoceno medio y tardío [tesis doctoral inédita. Universidad Nacional de La Plata].

Marani, H. y Borella, F. (2014). Patrón de explotación de tinámidos en la costa Atlántica Norpatagónica (Argentina) durante el Holoceno Tardío. Revista Chilena de Antropología, 29, 68-73.
Martínez, G. (2008-2009). Arqueología del Curso Inferior del Río Colorado: estado actual del conocimiento e implicaciones para la dinámica poblacional de cazadores-recolectores Pampeano-Patagónicos. Cazadores-recolectores del Cono Sur, 3, 71-92.

Martínez, G. (2015). Arqueología y pobladores antiguos de la cuenca del río Colorado. En M. Sili, A. Kozel y R. Bustos (Eds.), La Región del Colorado. Historia, cultura y paisaje en la frontera (pp. 28-47). Fundación ArgenINTA.

Mondini, M. (2002). Formación del registro arqueofaunístico en abrigos rocosos de la Puna Argentina. Tafonomía de carnívoros [tesis doctoral inédita. Universidad de Buenos Aires].

Montalvo, C. (2002). Root traces in fossil bones from the Huayquerian (Late Miocene) faunal assemblage of Telén, Argentina. Acta Geológica Hispánica, 37(1), 37-42.

Montalvo, C., Fernández, F. J., Tomassini, R., Mignino, J., Kin, M. y Santillán, M. (2020). Spatial and temporal taphonomic study of bone accumulations of the burrowing owl (Athene cunicularia) in central Argentina. Journal of Archaeological Science: Reports, 30, 1-11.

Musters, G. C. (1997 [1869-1970]). Vida entre los patagones. Un año de excursiones por tierras no frecuentadas desde el Estrecho de Magallanes hasta el Río Negro. El Elefante Blanco.

Narosky T. y Babarskas, M. (2001). Aves de la Patagonia. Guía para su reconocimiento. Vázquez Mazzini.

Olsen, S. y Shipman, P. (1988). Surface modification on bone: trampling versus butchery. Journal of Archaeological Science, 15(5), 535-553.

Politis, G. (2003). The theoretical landscape and the methodological development of archaeology in Latin America. American Antiquity, 68(2), 245-272.

Prates, L. (2008). Los indígenas del Río Negro. Un enfoque arqueológico. Sociedad Argentina de Antropología.

Prates, L. y Acosta Hospitaleche, C. (2010). Las aves de sitios arqueológicos del Holoceno tardío de Norpatagonia, Argentina. Archaeofauna, 19(1), 7-18.

Prates, L. y Bonomo, M. (2017). Los ambientes acuáticos en arqueología. Arqueología, 23(3), 11-33.

Quintana, C. (2008). Cálculo del número mínimo de individuos de huevos de ñandú. Intersecciones en Antropología, 9(9), 93-97.

Rufà, A., Blasco, R., Roger, T. y Moncel, M. (2015). What is the taphonomic agent responsible for the avian accumulation? An approach from the Middle and early Late Pleistocene assemblages from Payre and Abri des Pêcheurs (Ardèche, France). Quaternary International, 421, 46-61. 
Salemme, M. y Frontini, R. (2011). The exploitation of Rheidae in Pampa and Patagonia (Argentina) as recorded by chroniclers, naturalists and voyagers. Journal of Anthropological Archaeology, 30(4), 473-483.

Stoessel, L. y Alcaráz, A. P. (2017). Capítulo IV. Los conjuntos faunísticos. Subsistencia y tafonomía. En G. Martínez (Ed.), Arqueología de cazadores-recolectores del curso inferior del río Colorado (Provincia de Buenos Aires, Argentina). Aportes al conocimiento de las ocupaciones humanas Pampeano-Patagónicas (pp. 100-120). Serie Monográfica INCUAPA.

Stoessel, L. y Martínez, G. (2014). El proceso de intensificación en la transición pampeano-patagónica oriental: discusión y perspectivas comparativas con regiones aledañas. Comechingonia, 18(2), 65-94.

Stuiver, M., Reimer, P. y Reimer, R. (2017). CALIB7.1. http://calib.org (10 de julio 2019).
Svoboda, A. y Gómez Otero, J. (2015). Explotación de fauna dulceacuícola en el valle inferior del río Chubut (Patagonia central) durante el Holoceno tardío. Intersecciones en Antropología, 16(1), 39-52.

Tambussi, C. (1995). The fossil Rheiformes from Argentina. En D. Peters (Ed.), Acta palaeornithologica (pp. 121-

130). Forschungsinstitut Senckenberg.

Tonni, E. (1981). Restos de aves hallados en el Alero de los Sauces (Neuquén, Argentina). Trabajos de Prehistoria, 1, 89-92. 
